\begin{tabular}{|c|l|}
\hline Title & Eigenvector-based intergroup connection of low rank for hierarchical multi-agent dynamical systems \\
\hline Author(s) & Tsubakino, Daisuke; Hara, Shinji \\
\hline Citation & $\begin{array}{l}\text { Systems \& Control Letters, 61(2), 354.361 } \\
\text { https://doi.org/40.1016/.Sysconle.2011.11.008 }\end{array}$ \\
\hline Issue Date & 2012-02 \\
\hline Doc URL & http://hdl.handle.net/2115/48866 \\
\hline Type & article (author version) \\
\hline File Information & SCL61-2_354.361.pdf \\
\hline
\end{tabular}

Instructions for use 


\title{
Eigenvector-based intergroup connection of low rank for hierarchical multi-agent dynamical systems
}

\author{
Daisuke Tsubakinoa,*, Shinji Hara ${ }^{\mathrm{b}}$ \\ ${ }^{a}$ Division of Systems Science and Informatics, Hokkaido University, Kita 14, Nishi 9, Kita-ku, Sapporo, Hokkaido, 060-0814, Japan \\ ${ }^{b}$ Department of Information Physics and Computing, The University of Tokyo, 7-3-1 Hongo, Bunkyo, Tokyo, 113-8656, Japan
}

\begin{abstract}
This paper proposes an eigenvector-based method for analysis and design of hierarchical networks for multi-agent systems. We first define the concept of eigen-connection by characterizing low rank information flow between layers based on the eigenvector of lower level interconnection structures. It is shown that the resulting intergroup interconnections affect only a few eigenvalues of interconnection structures in the lower layer, and we derive explicit expressions for shifted eigenvalues. Then a procedure for designing hierarchical networks that result in desirable eigenvalue distributions is proposed, where the eigen-connection is used for a key to move undesirable eigenvalues selectively. The effectiveness of the procedure is demonstrated by a numerical example.
\end{abstract}

Keywords: multi-agent dynamical system, hierarchical network, low rank interconnection, eigenvector-based method

\section{Introduction}

Networked multi-agent dynamical systems are one of the classes of greatest concern in control engineering in recent years. A great number of researchers have paid attention to this field, especially consensus problems and cooperative control [1, 2, 3, 4]. Usually, eigenvalues of the matrix that represents the network structure play an important role in many application concerned with multi-agent systems. However, for systems with large-scale networks, it is extremely difficult to design an information protocol that results in a desirable eigenvalue distribution. In nature, it is often observed that an interaction, which seems to be large and complex from a global point of view, consists of a number of local interactions in small groups and weak interactions among the groups (see e.g. [5]). This hierarchical structure can be expected to be one of effective ways to handle systems with large scale network structure.

This is not the first attempt to introduce a hierarchy to network structures for multi-agent systems. For example, Smith et al. [6] proposed a hierarchical cyclic pursuit scheme and Hamilton and Broucke [7, 8] introduced a framework named patterned linear system which is capable of dealing with a class of hierarchy. This paper is related to [6], where agents, which are modeled as the integrator, are divided into some groups and the hierarchy means that cyclic pursuit is achieved both on a micro and a macro levels. That is, each agent pursues the next agent cyclically within a group and the centroid of each group also pursues that of the next group in the same manner. The scheme requires that an agent in a group receive information about two agents: the next agent in the group and a

\footnotetext{
${ }^{*}$ Corresponding author. Tel.:+81-11-706-6452

Email addresses: tsubakino@ssi.ist.hokudai.ac.jp (Daisuke Tsubakino), shinji_hara@ipc.i.u-tokyo.ac.jp (Shinji Hara)
}

corresponding agent in the next group. However, if we focus on information flow among groups, all information about agents in each group must be transmitted to the next groups. Hence, the proposed scheme does not seem to capture the weakness of intergroup connections.

Motivated by this fact, Shimizu and Hara generalized the hierarchical cyclic pursuit scheme and focused on an effect of the intergroup connection $[9,10,11]$. The difference from the previous scheme is the fashion of information exchange among the groups. In the newly proposed scheme, the only aggregated information about the agents in each group is transmitted to the next group. They related the aggregation to the matrix that appears in an off-diagonal block of the overall system matrix and regarded the strength of the intergroup connection as its rank. This new view leads to the concept of low rank interconnection. It is a realistic situation since the capacity of a communication channel is usually limited. Furthermore, it was shown that the low rank intergroup connections result in rapid convergence compared with the scheme in [6].

The superiority of the hierarchical schemes with low rank interconnection discussed by deriving explicit expressions of eigenvalues of the system matrix for hierarchical schemes. A remarkable fact is that eigenvalues are decomposed into some sets and members of each set coincide with eigenvalues of matrices representing local interconnection structures except a few eigenvalues. Interestingly, a similar result has been reported in [12], which employs a hierarchy in a study of vehicle formations. Whereas the network structure considered there is not a cyclic pursuit type, exchange of low rank information among groups is observed. Hence, we can expect that general hierarchical networks with low rank interconnections induce such specific eigenvalue distributions. If this is true, the property will give us an effective procedure for designing hierarchical network structures. 
The final goal of the present paper is to establish a framework for designing general hierarchical networks based on low rank properties. In particular, we concentrate on the fundamental case where each group contains the same number of agents as a first step. There are two main contributions in this paper, namely a new characterization of interlayer low rank information flow and an associated systematic design procedure for hierarchical multi-agent dynamical systems. Unfortunately, low rank interconnections do not always result in the specific eigenvalue distributions. Hence, an essential structure implicitly used in the previous works is clarified in this paper. It should be noticed that there is no trivial answer for this issue, since the explicit expressions of eigenvalues are derived by direct computation in the previous works.

As a solution of this problem, we first define a new class of low rank interlayer information flow based on eigenvectors of matrices corresponding to local network structure. It is shown that general hierarchical network structures together with intergroup connections belonging to the proposed class induce specific eigenvalue distributions. More precisely, intergroup connections in the proposed class affect only a few eigenvalues of local network structure. This completely explains the previous results in $[9,10,11,12]$. Furthermore, we can obtain explicit expressions describing how the corresponding eigenvalues are shifted. By utilizing this result, we next propose an efficient design method of hierarchical networks that result in desirable eigenvalue distributions. Briefly speaking, the proposed method is to design intergroup network so that undesirable eigenvalues of local interconnection structure are shifted selectively.

This paper is organized as follows. Section 2 introduces a general model of two-layer hierarchical multi-agent dynamical systems. In Section 3, a fundamental framework is developed for the rank one and the rank two cases. First, the concept of eigen-connection is defined. Then, we derive an expression for eigenvalue distributions of hierarchical network structures with eigen-connections. Section 4 investigates group behavior of agents over hierarchical networks with eigen-connections. Section 5 is devoted to a design procedure for hierarchical interconnections based on our framework. We will show that a low rank intergroup connection stabilizes unstable locallyconnected systems of dynamical agents by a numerical simulation. Some topics of extension to further general cases are discussed in Section 6.

Notation: The imaginary unit $\sqrt{-1}$ is denoted by i. For a square matrix $M, \sigma(M)$ denotes the set of all eigenvalues of $M . M^{\top}$ and $M^{*}$ represent the transpose and the conjugate transpose of $M$, respectively. $I_{d}$ is the $d \times d$ identity matrix and $\mathbf{1}_{d}$ is a $d$-dimensional column vector with all the components equal to one, that is, $\mathbf{1}_{d}=(1, \ldots, 1)^{\top} \in \mathbb{R}^{d}$.

\section{Hierarchical multi-agent dynamical systems}

We here introduce a general model for hierarchical multiagent dynamical systems that is investigated throughout this paper. The system consists of $N$ identical SISO agents whose state

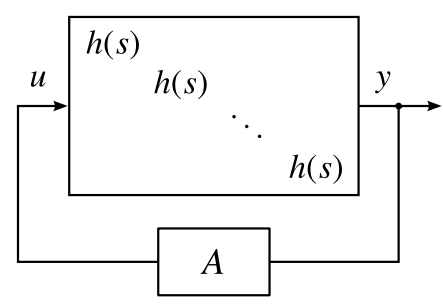

Figure 1: Interconnection structure.

space realization is expressed as

$$
\left\{\begin{array}{l}
\dot{x}_{i}=A_{h} x_{i}+b_{h} u_{i} \\
y_{i}=c_{h}^{\top} x_{i}
\end{array}, \quad i=1, \ldots, N\right.
$$

and the transfer function is given by

$$
h(s)=c_{h}^{\top}\left(s I_{n_{0}}-A_{h}\right)^{-1} b_{h},
$$

where $b_{h}, c_{h} \in \mathbb{R}^{n_{0}}$ and $A_{h} \in \mathbb{R}^{n_{0} \times n_{0}}$. The agents are connected to each other through the input and output according to the following rule:

$$
u=A y,
$$

where $y:=\left(y_{1}, \ldots, y_{N}\right)^{\top}, u:=\left(u_{1}, \ldots, u_{N}\right)^{\top}$ and $A \in \mathbb{R}^{N \times N}$. The situation is depicted in Fig. 1 . If the $i j$ th entry of $A$ is nonzero, the $i$ th agent receives output from the $j$ th agent. We refer to $A$ as an interconnection or an interconnection structure in the remaining part of the paper. The closed-loop system is then represented by

$$
\dot{x}=\mathcal{A} x, \quad x:=\left(\begin{array}{lll}
x_{1}^{\top} & \cdots & x_{N}^{\top}
\end{array}\right)^{\top},
$$

where $\mathcal{A} \in \mathbb{R}^{\left(n_{0} N\right) \times\left(n_{0} N\right)}$ is the system matrix of the total system defined by

$$
\mathcal{A}=I_{N} \otimes A_{h}+A \otimes\left(b_{h} c_{h}^{\top}\right) .
$$

Stability of the closed-loop system is completely determined by eigenvalues of $\mathcal{A}$. Massioni and Verhaegen proposed a procedure to design distributed controllers for systems that have a similar structure to (2) in [13]. Unlike this work, we would like to design $A$ so that the eigenvalue distribution of $\mathcal{A}$ becomes desirable. This is not an easy task in direct methods, especially when $N$ is very large. Fortunately, the closed-loop system belongs to the class of LTI systems with generalized frequency variables [14]. Thus, we only have to check if all the eigenvalues of $A$ (not, $\mathcal{A})$ lie on the associated stability region determined by $h(s)$. Note that two types of necessary and sufficient stability condition, namely Hurwitz type and Lyapunov type, in terms of the coefficients of $h(s)$ were derived in [15].

As mentioned in Introduction, we consider hierarchical interconnection structures in this paper. Let agents be divided into $n_{2}$ groups including $n_{1}$ agents, where $N=n_{1} n_{2}$. The augmented state $x$ is parted as follows:

$$
x=\left(\begin{array}{llll}
X_{1}^{\top} & X_{2}^{\top} & \cdots & X_{n_{2}}^{\top}
\end{array}\right)^{\top},
$$

where $X_{i_{2}}:=\left(x_{\left(i_{2}-1\right) n_{1}+1}^{\top}, \cdots, x_{i_{2} n_{1}}\right)^{\top}$ is the state of the $i_{2}$ th group. A two-layer hierarchical interconnection structure is given by

$$
A=I_{n_{2}} \otimes A_{1}+K_{2} \otimes \Delta_{1},
$$




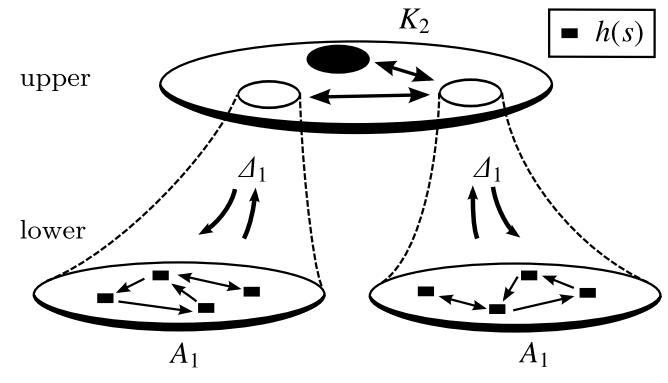

Figure 2: Two-layer hierarchically interconnected system.

where $A_{1}, \Delta_{1} \in \mathbb{R}^{n_{1} \times n_{1}}$ and $K_{2} \in \mathbb{R}^{n_{2} \times n_{2}}$. Figure 2 illustrates the network structure schematically. Agents locally interact with each other in each group. The hierarchy means that the groups also interact with each other in the upper layer. The matrices $A_{1}$ and $K_{2}$ are models of the local interaction in the lower layer and the intergroup interaction in the upper layer, respectively. Hence, non-zero entries of $K_{2}$ indicate the existence of communication between the corresponding groups. The matrix $\Delta_{1}$ determines what kind of information of each group is exchanged among the groups and which agents receive the effect of the interaction in the upper layer. In other words, $\Delta_{1}$ models interlayer information flow. We refer to $\Delta_{1}$ as an intergroup connection matrix or, simply, a connection matrix.

The connection matrix $\Delta_{1}$ clearly plays an important role in the hierarchical interconnection structure. In particular, if agents have little interaction with the others in each group and the resulting matrix $A_{1}$ is sparse, the group interaction dominates the total behavior. As a result, a choice of the matrix $\Delta_{1}$ is significant for the achievement of global objectives. Shimizu and Hara claimed that the rank of $\Delta_{1}$ captures a degree of aggregation of information flow among the group $[9,10]$. We follow the idea of focusing on the low rank property of $\Delta_{1}$. A problem considered in this paper is how we can utilize $\Delta_{1}$ in the design of a hierarchical interconnection $A$ with a desirable eigenvalue distribution. The key feature is to characterize $\Delta_{1}$ based on the eigenvector of $A_{1}$.

\section{Eigenvalue distribution}

In this section, we define a class of intergroup connection matrices based on the eigenvector of the local interconnection. This new characterization allows us to obtain analytical eigenvalue distribution of the resulting hierarchical interconnection. We mainly focus on the rank one and rank two cases. However, the extensions to higher rank cases are systematically possible.

For convenience of analysis, all matrices and vectors are allowed to have complex entries in this section. However, results below are still valid for real matrices and vectors.

\subsection{Rank one interconnection}

Consider rank one interconnections. That is, we have

$$
\operatorname{rank} \Delta_{1}=1
$$

Without loss of generality, we can express $\Delta_{1}$ as the product of two vectors:

$$
\Delta_{1}=\mu \zeta^{*}
$$

where $\mu, \zeta$ are $n_{1}$-dimensional column vectors. Note that this decomposition is unique up to scalar multiplication. The above form tells us that $\zeta^{*}$ and $\mu$ represent ways of aggregation and distribution of information, respectively. In what follows, we characterize $\Delta_{1}$ by eigenvectors of $A_{1}$.

Definition 1. Let $A_{1}$ be an $n_{1} \times n_{1}$ matrix that has an eigenvalue $\lambda_{1}$. An intergroup connection matrix defined by (4) is a left (resp., right) eigen-connection matrix of $A_{1}$ associated with the eigenvalue $\lambda_{1}$, if $\zeta$ (resp., $\mu$ ) is a left (resp., right) eigenvector of $A_{1}$ associated with the eigenvalue $\lambda_{1}$.

One may think that the above definition is somewhat artificial. However, if $A_{1}$ is a graph Laplacian and $\zeta=\left(1 / n_{1}\right) \mathbf{1}_{n_{1}}$, then the resulting intergroup connection matrix $\Delta_{1}$ is an eigenconnection matrix of $A_{1}$. This setting corresponds to the case where group averages are exchanged among the groups in the upper layer and it is a quite natural situation. With this new characterization of intergroup connection matrices, we can derive the following theorem that shows the eigenvalue distribution of $A$ can be decomposed into two set. This is the first feature of this paper.

Theorem 1. Let $A_{1}$ be an $n_{1} \times n_{1}$ matrix that has at least one simple eigenvalue $\lambda_{1}$ and let $\mu$ and $\zeta$ be $n_{1}$-dimensional column vectors. If $\Delta_{1}=\mu \zeta^{*}$ is a left or a right eigen-connection matrix of $A_{1}$ associated with $\lambda_{1}$, then, for any $n_{2} \times n_{2}$ matrix $K_{2}$, the set of all the eigenvalues of $A$ defined by (3) is given by

$$
\sigma(A)=\left\{\lambda_{1}+\gamma \zeta^{*} \mu \mid \gamma \in \sigma\left(K_{2}\right)\right\} \cup\left(\sigma\left(A_{1}\right) \backslash\left\{\lambda_{1}\right\}\right),
$$

Furthermore, if $\lambda_{i} \in \sigma\left(A_{1}\right) \backslash\left\{\lambda_{1}\right\}$ is an $m_{i}$ times repeated eigenvalue of $A_{1}$, then $\lambda_{i}$ has algebraic multiplicity $n_{2} m_{i}$.

Proof. See Appendix A.

Theorem 1 tells us that an intergroup eigen-connection matrix of rank one affects only one eigenvalue of the local interconnection $A_{1}$. In the previous research [9], $A_{1}$ is a Laplacian matrix, $\zeta$ is an arbitrary stochastic vector, and $\mu=\mathbf{1}_{n_{1}}$. This condition corresponds to the case where the resulting intergroup connection matrix $\Delta_{1}$ is a right eigen-connection because the Laplacian matrix always has $\mathbf{1}_{n_{1}}$ as a right eigenvector associated with an eigenvalue 0 . This means that the previous result in [9] is led by the fact that $\Delta_{1}$ is not only a matrix of rank one but also an eigen-connection matrix. Actually, general rank one intergroup connection matrices do not always affect only one eigenvalue of $A_{1}$. The theorem also contains the result shown in [12]. In their research, the matrix $A_{1}$ is the Laplacian matrix whose entries in the first row are all zero and $\Delta_{1}$ is the matrix that has a non-zero number only at $(1,1)$ entry. The matrix $\Delta_{1}$ can be expressed by $\Delta_{1}=e_{1}^{n_{1}} e_{1}^{n_{1} \top}$, where $e_{1}^{n_{1}}$ is a vector $(1,0, \ldots, 0)^{\top} \in \mathbb{R}^{n_{1}}$. Obviously, $\Delta_{1}$ is a left eigen-connection matrix of $A_{1}$ associated with an eigenvalue 0 .

Theorem 1 has another feature. It enables us to design eigenvalue distribution of $A$ explicitly by adjusting $A_{1}, K_{2}$, and $\Delta_{1}$. 
This means that we can develop a global interconnection structure from local ones. This is an advantage to introduce a hierarchical structure with low rank eigen-connection. In particular, the term $\gamma \zeta^{*} \mu$ is important. If $\gamma \zeta^{*} \mu$ does not change, no change of eigenvalue distribution occurs even though $K_{2}$ and $\Delta_{1}$ may change. We will propose an design procedure of $A$ by using this property in Section 5.

A problem that arises in an application is that we can not always choose $\zeta$ from the eigenvectors of $A_{1}$. Furthermore, the corresponding eigenvector may be a complex vector. Although the theorem holds even if $\zeta$ is a complex vector, $\zeta$ must be a real vector for practical reasons. One of the methods to overcome such a situation is to choose $\zeta$ from linear combinations of eigenvectors of $A_{1}$. Actually, this is a special case of a higher rank eigen-connection. The details will be discussed in the next section. Hence, we move on to the rank two case without discussing this topic here.

\subsection{Rank two interconnection}

The concept of eigen-connection, which is introduced in the previous subsection, can be naturally extended to the rank two case. In this subsection, we define a class of eigen-connection matrices for intergroup connection matrices of rank two and prove a theorem similar to Theorem 1 . Since rank $\Delta_{1}=2$, we can decompose $\Delta_{1}$ into a product of two $2 \times n_{1}$ matrices:

$$
\Delta_{1}=\left(\begin{array}{ll}
\mu_{1} & \mu_{2}
\end{array}\right)\left(\begin{array}{ll}
\zeta_{1} & \zeta_{2}
\end{array}\right)^{*}
$$

In contrast to the rank one case, this decomposition is not unique since there is a degree of freedom of the linear combination. We define the eigen-connection of rank two by taking into account this freedom.

Definition 2. Let $A_{1}$ be an $n_{1} \times n_{1}$ matrix and let $\lambda_{1}$ and $\lambda_{2}$ be two eigenvalues of $A_{1}$. An intergroup connection matrix given by (5) is a left (resp., right) eigen-connection matrix of $A_{1}$ associated with the eigenvalues $\lambda_{1}$ and $\lambda_{2}$, if $\zeta_{1}$ and $\zeta_{2}$ (resp., $\mu_{1}$ and $\mu_{2}$ ) are linearly independent and belong to the linear subspace spanned by the left (resp., right) eigenvectors associated with the eigenvalues $\lambda_{1}$ and $\lambda_{2}$.

The above definition is a natural extension of the rank one case. Note that if an intergroup connection matrix $\Delta_{1}$ given by (5) is a left (resp., right) eigen-connection of $A_{1}$, there exist a $2 \times 2$ matrix $T$ (resp., $S$ ) such that

$$
\left(\begin{array}{ll}
\zeta_{1} & \zeta_{2}
\end{array}\right)=\left(\begin{array}{ll}
v_{1} & v_{2}
\end{array}\right) T, \quad\left(\text { resp., }\left(\begin{array}{ll}
\mu_{1} & \mu_{2}
\end{array}\right)=\left(\begin{array}{ll}
w_{1} & w_{2}
\end{array}\right) S\right),
$$

where $v_{1}$ and $v_{2}$ (resp., $w_{1}$ and $w_{2}$ ) are left (resp., right) eigenvectors of $A_{1}$. We show the main result for hierarchical interconnections with eigen-connections of rank two.

Theorem 2. Let $A_{1}$ be an $n_{1} \times n_{1}$ matrix that has two simple eigenvalues $\lambda_{1}$ and $\lambda_{2}$ and let $\mu_{1}, \mu_{2}, \zeta_{1}$, and $\zeta_{2}$ be $n_{1}$ dimensional column vectors. If $\Delta_{1}$ given by (5) is a left eigenconnection of $A_{1}$ associated with $\lambda_{1}$ and $\lambda_{2}$, then, for any $n_{2} \times n_{2}$ matrix $K_{2}$, the set of all the eigenvalues of A defined by (3) is given by

$$
\sigma(A)=\left(\bigcup_{\gamma \in \sigma\left(K_{2}\right)} \sigma\left(\Phi_{\gamma}\right)\right) \cup\left(\sigma\left(A_{1}\right) \backslash\left\{\lambda_{1}, \lambda_{2}\right\}\right) .
$$

Here, for a complex value $\gamma, \Phi_{\gamma}$ is a $2 \times 2$ matrix defined by

$$
\Phi_{\gamma}:=\left(\begin{array}{ll}
\lambda_{1} & \\
& \lambda_{2}
\end{array}\right)+\gamma\left(\begin{array}{l}
v_{1}^{*} \\
v_{2}^{*}
\end{array}\right)\left(\begin{array}{ll}
\mu_{1} & \mu_{2}
\end{array}\right) T^{*},
$$

where $v_{1}$ and $v_{2}$ are the left eigenvectors associated with $\lambda_{1}$ and $\lambda_{2}$ and $T$ is a $2 \times 2$ matrix satisfying

$$
\left(\begin{array}{ll}
\zeta_{1} & \zeta_{2}
\end{array}\right)=\left(\begin{array}{ll}
v_{1} & v_{2}
\end{array}\right) T .
$$

Proof. See Appendix B.

As in the rank one case, we can obtain an analogous result for right eigen-connection matrices.

Corollary 1. In the same setting in Theorem 2, if $\Delta_{1}$ is a right eigen-connection matrix of $A$ associated with $\lambda_{1}$ and $\lambda_{2}$, then the same statement of Theorem 2 holds by replacing $\Phi_{\gamma}$ by

$$
\Psi_{\gamma}:=\left(\begin{array}{ll}
\lambda_{1} & \\
& \lambda_{2}
\end{array}\right)+\gamma S\left(\begin{array}{l}
\zeta_{1}^{*} \\
\zeta_{2}^{*}
\end{array}\right)\left(\begin{array}{ll}
w_{1} & w_{2}
\end{array}\right),
$$

where $w_{1}$ and $w_{2}$ are the right eigenvectors of $A_{1}$ associated with $\lambda_{1}$ and $\lambda_{2}$ and $S$ is a $2 \times 2$ matrix satisfying

$$
\left(\begin{array}{ll}
\mu_{1} & \mu_{2}
\end{array}\right)=\left(\begin{array}{ll}
w_{1} & w_{2}
\end{array}\right) S
$$

Note that the above theorem does not depend on the choice of $\mu_{1}$ and $\mu_{2}$ and that its corollary does not depend on $\zeta_{1}$ and $\zeta_{2}$, either. We can conclude that intergroup connection matrices of rank two can change at least two eigenvalues of $A_{1}$. Unlike the rank one case, the resulting eigenvalues are not explicitly obtained even if the connection matrix is a rank two eigenconnection matrix. They are, however, given as the eigenvalues of a $2 \times 2$ matrix, which can be easily calculated.

This result covers the previous result in [10] as a special case. In their setting, $\mu_{1}$ and $\mu_{2}$ are the sum and the difference of two eigenvectors of $A_{1}$ by chance. Thus, $\Delta_{1}$ is a right eigenconnection matrix of $A_{1}$. In comparison with the previous research, our characterization of intergroup connection matrix of rank two is applicable to any class of $A_{1}$ and $K_{2}$. Besides, an expression of the $2 \times 2$ matrix that determines the shifted eigenvalues is explicitly obtained.

\section{Group behavior}

In Section 3, we showed that the eigenvalue distribution of a hierarchical interconnection structure with a low rank eigenconnection matrix is divided into two parts. We here relate this structure to behavior of each group.

Consider the rank one case, that is, $\Delta_{1}$ is defined by (4). Assume that $\Delta_{1}$ is a left eigen-connection matrix of $A_{1}$ associated 
with a simple eigenvalue $\lambda_{1}$. We also assume that $\zeta$ is not orthogonal to $\mu$, that is, $\zeta^{\top} \mu \neq 0$, to prevent $\Delta_{1}$ from being a nilpotent matrix. For each group, we define the representative state $Y_{i_{2}}(t) \in \mathbb{R}^{n_{0}}, i_{2}=1, \ldots, n_{2}$ by

$$
Y_{i_{2}}=\sum_{i_{1}=1}^{n_{1}} \zeta_{i_{1}} x_{\left(i_{2}-1\right) n_{2}+i_{1}}=\left(\zeta^{\top} \otimes I_{n_{0}}\right) X_{i_{2}},
$$

where $\zeta_{i_{1}}\left(i_{1}=1, \ldots, n_{1}\right)$ is the $i_{1}$ th component of $\zeta$ and $X_{i_{2}}$ is the state of $i_{2}$ th group. Namely, $Y_{i_{2}}$ is a weighted sum of the agents' state in the $i_{2}$ th group. The collection of all $Y_{i_{2}}$, which is denoted by $Y$, can be written as

$$
Y=\left(\begin{array}{lll}
Y_{1}^{\top} & \cdots & Y_{n_{2}}^{\top}
\end{array}\right)^{\top}=\left(I_{n_{2}} \otimes \zeta^{\top} \otimes I_{n_{0}}\right) x .
$$

We now investigate the time rate of change of $Y$ to clarify why the word representative is used.

Let $P$ be an $n_{1} \times n_{1}$ matrix defined by

$$
P:=\left(\begin{array}{llll}
\zeta & v_{2} & \cdots & v_{n_{1}}
\end{array}\right)^{\top}
$$

where $v_{2}, \ldots, v_{n_{1}}$ are $n_{1}-1$ linearly independent vectors orthogonal to $\mu$. From the assumption that $\zeta^{\top} \mu \neq 0, P$ is non-singular and its inverse has the form

$$
P^{-1}=\left(\begin{array}{llll}
\left(\zeta^{\top} \mu\right)^{-1} \mu & w_{2} & \cdots & w_{n_{1}}
\end{array}\right)
$$

for some appropriate vectors $w_{2}, \ldots, w_{n_{1}}$. It follows immediately from the definition that

$$
P A_{1} P^{-1}=\left(\begin{array}{c|c}
\lambda_{1} & \\
\hline * & *
\end{array}\right), \quad P \Delta_{1} P^{-1}=\left(\begin{array}{l|l}
\zeta^{\top} \mu & \\
\hline &
\end{array}\right),
$$

where empty blocks mean that all the entries are 0 . Next, consider the coordinate transformation $x \mapsto z=\mathcal{T} x$, where $\mathcal{T}$ is defined by

$$
\mathcal{T}:=I_{n_{2}} \otimes P \otimes I_{n_{0}}
$$

Note that the transformation by the above matrix preserves the hierarchical structure shown in Fig. 2. Then, $Y$ satisfies the following relation:

$$
Y=\left(I_{n_{2}} \otimes\left(\begin{array}{llll}
1 & 0 & \cdots & 0
\end{array}\right) \otimes I_{n_{0}}\right) z
$$

Since the transformed system matrix is represented by

$$
\begin{aligned}
\mathcal{T} \mathcal{A} \mathcal{T}^{-1}= & I_{n_{2}} \otimes I_{n_{1}} \otimes A_{h}+I_{n_{2}} \otimes\left(P A_{1} P^{-1}\right) \otimes\left(b_{h} c_{h}^{\top}\right) \\
& +K_{2} \otimes\left(P A_{1} P^{-1}\right) \otimes\left(b_{h} c_{h}^{\top}\right),
\end{aligned}
$$

substituting (7) into the above equation and left-multiplying by the matrix in (8) yield

$$
\dot{Y}=\left(I_{n_{2}} \otimes A_{h}+\left(\lambda_{1} I_{n_{2}}+\left(\zeta^{\top} \mu\right) K_{2}\right) \otimes\left(b_{h} c_{h}^{\top}\right)\right) Y .
$$

This implies that the representative state evolves by itself. Compared with (1)-(2), this is an interconnected system of identical $n_{2}$ agents that have the same state space realization $\left(A_{h}, b_{h}, c_{h}^{\top}\right)$ as that of the original agents (see Fig. 3). We can regard the representative state $Y_{i_{2}}$ as the state of the leader of $i_{2}$ th group. As is clear from (7), this virtual leader communicates only with

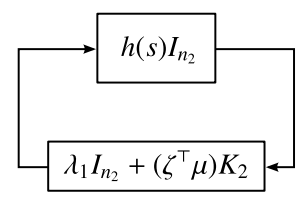

Figure 3: Feedback configuration of the representative state.

the other leaders. The other agents in each group interact with the agents in the other groups through the leader. If $\lambda_{1}=0$ and $K_{2}$ is a Laplacian matrix, the situation corresponds to that in [12]. Hence, we can conclude that a hierarchical interconnection structure with the rank one eigen-interconnection implicitly assigns a virtual leader in each group. Since the interconnection structure of leaders is given by $\lambda_{1} I_{n_{2}}+\left(\zeta^{\top} \mu\right) K_{2}$, the first set of the eigenvalues of $A$ in Theorem 1 is related with the behavior of leaders.

In the case where $\Delta_{1}$ is given by (5), a similar result is available under the same condition as in Theorem 2 . The number of the virtual leaders in each group is two in this case.

\section{Design procedure}

We here show our procedure for designing hierarchical networks for multi-agent dynamical systems based on the eigenconnection that has been developed in Section 3. The proposed procedure is examined by a numerical simulation.

\subsection{Procedure based on eigen-connections}

In control of multi-agent dynamical systems, an information exchange protocol among agents must be designed appropriately so that the corresponding interconnection structure $A$ has a desirable eigenvalue distribution. As seen in Section 3, eigenconnections can move eigenvalues of a local interconnection structure $A_{1}$ selectively. Assume that $A_{1}$ has an undesirable eigenvalue. Then, we can move it by letting $\mu$ or $\zeta$ be the corresponding eigenvector. If $A_{1}$ has two undesirable eigenvalues, eigen-connections of two rank are available to move them. Hence, we propose the following procedure for designing a hierarchical interconnection structure:

1. design the local interconnection structure $A_{1}$,

2. identify undesirable eigenvalues of $A_{1}$,

3 . construct connection matrix $\Delta_{1}$ based on the corresponding eigenvectors, and

4. design intergroup interconnection structure $K_{2}$ and adjust $\Delta_{1}$ so that shifted eigenvalues become desirable.

It should be noted that our procedure is applicable in the case where $A_{1}$ has more than two undesirable eigenvalues along with eigen-connections of higher rank as we shall discuss in the next section. In what follows, we demonstrate the above procedure by a numerical simulation. 


\subsection{Numerical simulation}

Consider the cooperative stabilization of 50 agents (i.e. $N=$ 50) whose transfer function is given by $h(s)=2 /\left(s^{3}+s^{2}+5 s\right)$. According to the stability analysis of LTI systems with generalized frequency variables [14], the augmented system matrix $\mathcal{A}$ given by (2) is stable if and only if all the eigenvalues of interconnection $A$ lie in $\Omega_{+}^{c}$. Here, $\Omega_{+}^{c}$ is the complement of $\Omega_{+}$ in $\mathbb{C}$, that is $\Omega_{+}^{c}=\mathbb{C} \backslash \Omega_{+}$and $\Omega_{+}$is defined as follows. Let $\phi(s):=1 / h(s)$ and $\mathbb{C}_{+}=\{s \in \mathbb{C} \mid \operatorname{Re} s \geq 0\}$. Then the region $\Omega_{+}$ is defined by

$$
\Omega_{+}:=\phi\left(\mathbb{C}_{+}\right)=\left\{\lambda \in \mathbb{C} \mid \exists s \in \mathbb{C}_{+} \text {such that } \phi(s)=\lambda\right\} .
$$

Thus, we must design a $50 \times 50$ matrix $A$ that has all the eigenvalues in $\Omega_{+}^{c}$.

In this example, we divide agents into 10 groups containing 5 agents each (i.e. $n_{2}=10, n_{1}=5$ ) to design a hierarchical interconnection. The first task in our procedure is the design of $A_{1}$. Let the local interconnection structure $A_{1}$ be given by

$$
A_{1}=\left(\begin{array}{rrrrr}
-1 & 0 & 0 & 1 & 0 \\
1 & -1 & 0 & 0 & 0 \\
0 & 1 & -2 & 1 & 0 \\
0 & 0 & 1 & -2 & 1 \\
1 & 1 & 0 & 0 & -2
\end{array}\right) .
$$

This $A_{1}$ corresponds to the communication topology shown in Fig. 4. All the eigenvalues of $A_{1}$ are plotted in Fig. 5 together with the stability region generated by $h(s)$. If no interaction occurs among the groups, that is, $\Delta_{1}=0$ or $K_{2}=0$, the resulting interconnection structure becomes $A=I_{n_{2}} \otimes A_{1}$. Hence, one may expect that the augmented system is stable because all the eigenvalues of $A$ are the same as $A_{1}$ and they are in the left half plane of complex plane. However, this is not true. There are two eigenvalues 0 and -3 that do not belong to $\Omega_{+}^{c}$. Thus, we design $\Delta_{1}$ and $K_{2}$ so that the unstable eigenvalues 0 and -3 are shifted into $\Omega_{+}^{c 1}$.

To this end, we next construct $\Delta_{1}$ based on eigenvectors of $A_{1}$ associated with the unstable eigenvalue 0 and -3 . Right eigenvectors of $A_{1}$ associated with 0 and -3 are

$$
w_{1}=\left(\begin{array}{lllll}
1 & 1 & 1 & 1 & 1
\end{array}\right)^{\top}, \quad w_{2}=\left(\begin{array}{lllll}
-2 & 1 & -5 & 4 & 1
\end{array}\right)^{\top},
$$

respectively. Now we set

$$
S=\frac{1}{3}\left(\begin{array}{rr}
2 & 1 \\
1 & -1
\end{array}\right)
$$

which leads to

$$
\left(\begin{array}{ll}
\mu_{1} & \mu_{2}
\end{array}\right)=\left(\begin{array}{ll}
w_{1} & w_{2}
\end{array}\right) S=\left(\begin{array}{rrrrr}
0 & 1 & -1 & 2 & 1 \\
1 & 0 & 2 & -1 & 0
\end{array}\right)^{\top} .
$$

The vectors $\zeta_{1}$ and $\zeta_{2}$ are chosen as

$$
\zeta_{1}=\frac{1}{3}\left(\begin{array}{lllll}
0 & 1 & 1 & 0 & 1
\end{array}\right)^{\top}, \quad \zeta_{2}=\frac{1}{3}\left(\begin{array}{lllll}
0 & 1 & 0 & 1 & 1
\end{array}\right)^{\top} .
$$

\footnotetext{
${ }^{1}$ Note that this is not the only method of stabilization. Actually, modification of $h(s)$ or $A_{1}$ allows the augmented system to be stable.
}

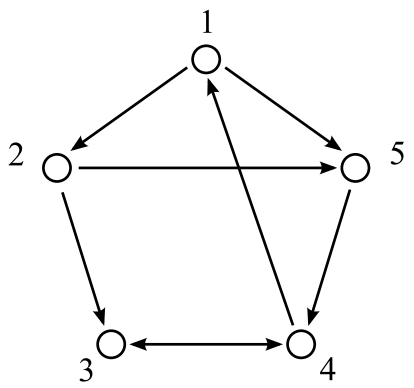

Figure 4: Local communication topology.

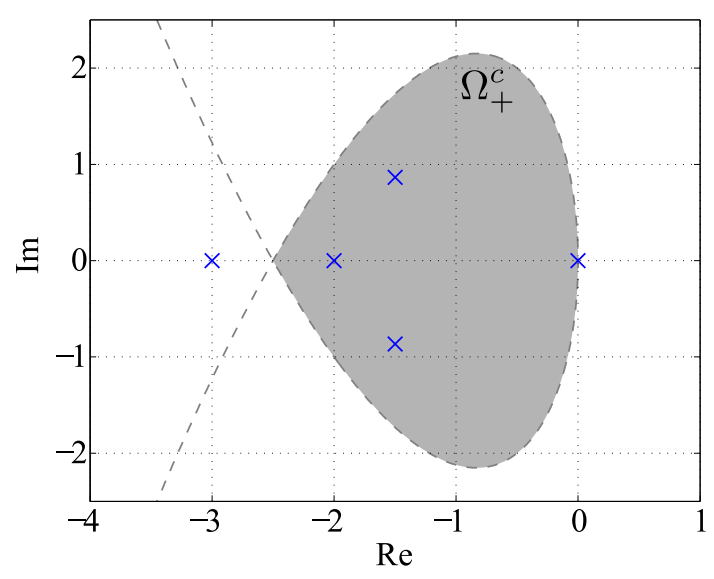

Figure 5: Eigenvalue distribution of $A_{1}$ and stability region $\Omega_{+}^{c}$ generated by $h(s)$.

It follows from the definition that the resulting $\Delta_{1}$ is a rank two right eigen-connection matrix of $A_{1}$ associated with 0 and -3 . The situation can be explained intuitively as follows. Two kinds of aggregated information are shared among the groups. The former, which is determined by $\zeta_{1}$, is the average output of the second, the third, and the fifth agents in each group. An effect of the intergroup interaction based on this aggregated information is transmitted to agents in each group according to $\mu_{1}$. In this case, all the agents except the first one receive it. On the other hand, the latter is the average output of the second, the fourth, and the fifth agents. In each group, the first, the third, and the fourth agents receive an effect of the intergroup interaction based on the aggregated information of second kind.

We apply Corollary 1 to compute eigenvalues of the total interconnection structure. Simple computation yields the following expression of $\Psi_{\gamma}$ :

$$
\Psi_{\gamma}=\left(\begin{array}{cc}
\gamma & 0 \\
0 & -3-\gamma
\end{array}\right)
$$

Hence, we have $\sigma\left(\Psi_{\gamma}\right)=\{\gamma,-3-\gamma\}$. The remaining task in our procedure is to design $K_{2}$ such that for each eigenvalue $\gamma$ of $K_{2}, \gamma$ and $-3-\gamma$ belong to $\Omega_{+}^{c}$. The following $K_{2}$ satisfies the requirement:

$$
K_{2}=-\frac{1}{4} L_{2}-\frac{5}{4} I_{10}
$$




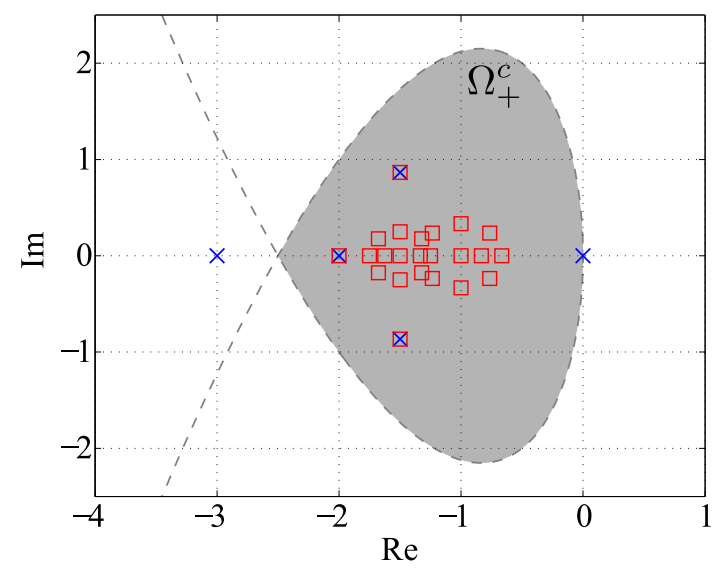

Figure 6: Eigenvalue distributions of $A$ (' $\square$ ') and $A_{1}$ ('×’).

where a $10 \times 10$ matrix $L_{2}$ is defined by

$$
L_{2}=\left(\begin{array}{rrrrrr}
1 & -1 & & & & \\
& \ddots & \ddots & & & \\
& & \ddots & -1 & & \\
-1 & & & 1 & & \\
& & & -1 & 1 & \\
& & & -1 & -1 / 2 & 3 / 2
\end{array}\right) .
$$

This means that 8 groups are in cyclic pursuit but the rest are not. Consequently, the network structure in the upper layer is not a cyclic type.

The resulting eigenvalue distribution of $A$ is plotted with ' $\square$ ' in Fig. 6. All the eigenvalues of $A$ are included in $\Omega_{+}^{c}$. Thus, we have accomplished our objective. We also plot the eigenvalues of $A_{1}$ with ' $\times$ ' for comparison. It is easily seen that only 0 and -3 are moved with the rest of the eigenvalues of $A_{1}$ remaining. This is one of the notable properties of hierarchical systems with eigen-connection of low rank. Initial condition responses of all agents are shown in Fig. 7. A point we should emphasize is that, even though the local interconnection is unstable, the outputs of all the agents converge to zero thanks to a good combination of hierarchical interconnection.

\section{Further general interconnections}

There are some directions of extension of the results in the previous sections. We briefly comment on them in this section. The first is to increase the rank of the intergroup connection matrix. The extension is more or less straightforward. A rank $r$ intergroup eigen-connection would change $r$ eigenvalues of $A_{1}$ and the affected eigenvalues would be given as the eigenvalues of a certain $r \times r$ matrix. An increase in dimension of matrices makes it difficult to calculate the resulting eigenvalue distribution. Thus, such an extension is not necessarily useful. We can interpret the result in [6] based on the eigen-connection ${ }^{2}$. Any

\footnotetext{
${ }^{2}$ Normally, it is interpreted as a property of Kronecker sum [16].
}

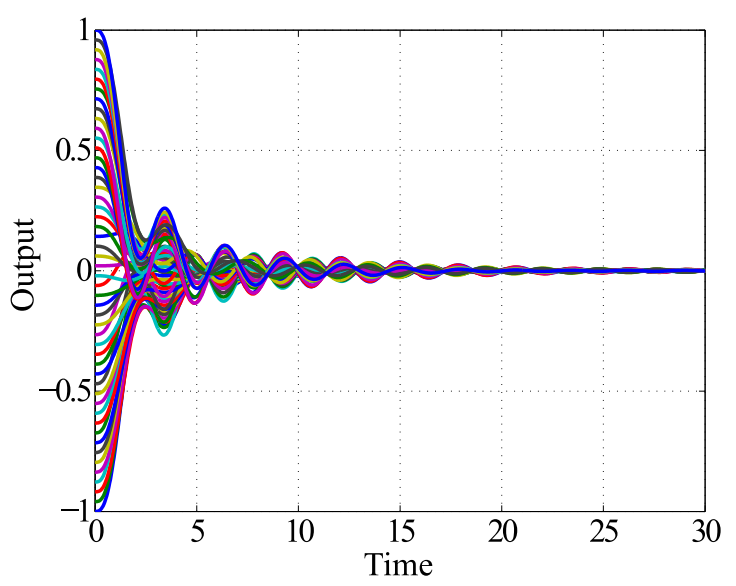

Figure 7: Outputs of all agents.

vector in $\mathbb{R}^{n_{1}}$ is an eigenvector of $I_{n_{1}}$. Thus, $A_{1}$ and $I_{n_{1}}$ have all the eigenvectors in common. This means $I_{n_{1}}$ is a full rank eigen-connection matrix of $A_{1}$ and all the eigenvalues of $A_{1}$ are affected.

The second extension is to increase the number of eigenvectors of $A_{1}$ that consist intergroup connection matrices without increasing the rank. Consider the rank one case. Let $\zeta$ be a linear combination of two eigenvectors of $A_{1}$, that is, there exist two constants $a_{1}$ and $a_{2}$ such that

$$
\zeta=a_{1} v_{1}+a_{2} v_{2}
$$

holds, where $v_{1}$ and $v_{2}$ are eigenvectors of $A_{1}$ associated with two distinct eigenvalues. Note that $v_{1}$ and $v_{2}$ are not necessary real even if $\zeta$ is a real vector. Without loss of generality, we can set $a_{1}=a_{2}=1$ since the scalar multiple of an eigenvector remains to be an eigenvector. The resulting intergroup connection matrix $\Delta_{1}$ is given by

$$
\Delta_{1}=\mu \zeta^{*}=\mu\left(\begin{array}{ll}
1 & 1
\end{array}\right)\left(\begin{array}{l}
v_{1}^{*} \\
v_{2}^{*}
\end{array}\right)=\left(\begin{array}{ll}
\mu & \mu
\end{array}\right)\left(\begin{array}{l}
v_{1}^{*} \\
v_{2}^{*}
\end{array}\right) .
$$

The matrix $\Delta_{1}$ is of the same form as a rank two intergroup connection matrix (5), although the rank of $\Delta_{1}$ is one. This means that the rank one intergroup connection matrix written as the product of an arbitrary vector and a linear combination of two eigenvectors of $A_{1}$ is equivalent to a degenerate rank two eigen-connection matrix. Thus, we can apply Theorem 2 to such matrices if the associated eigenvalues are simple. The matrix $T$ in (6) is always the second order identity matrix $I_{2}$ in this case. Thus, (6) can be written as

$$
\Phi_{\gamma}=\left(\begin{array}{cc}
\lambda_{1}+\gamma v_{1}^{*} \mu & \gamma v_{1}^{*} \mu \\
\gamma v_{2}^{*} \mu & \lambda_{2}+\gamma v_{2}^{*} \mu
\end{array}\right) .
$$

This is a simple $2 \times 2$ matrix, thus we can calculate the eigenvalues as follows:

$$
\begin{aligned}
& \frac{\lambda_{1}+\lambda_{2}+\gamma\left(\zeta^{*} \mu\right)}{2} \\
& \pm \frac{\sqrt{\left(\lambda_{1}-\lambda_{2}+\gamma\left(v_{1}-v_{2}\right)^{*} \mu\right)^{2}+4 \gamma^{2}\left(v_{1}^{*} \mu\right)\left(v_{2}^{*} \mu\right)}}{2} .
\end{aligned}
$$


It is clear that the resulting eigenvalues depend on the sum, difference and product of two quantity $\gamma v_{1}^{*} \mu$ and $\gamma v_{2}^{*} \mu$. This fact gives an index to design desirable eigenvalue distribution. To treat linear combinations of an arbitrary number of eigenvectors of $A_{1}$, we need results for higher rank interconnections, which is mentioned in the previous paragraph. Therefore, those two extensions are essentially same.

The final possibility of extensions is to increase the number of intergroup connections. If we employ $m$ connection matrices, the total interconnection structure is given by

$$
A=I_{n_{2}} \otimes A_{1}+\sum_{k=1}^{m} K_{2}^{(k)} \otimes \Delta_{1}^{(k)} .
$$

It is immediate from the proofs of Theorem 1 and Theorem 2 that similar results can be obtained if $K_{2}^{(1)}, \ldots, K_{2}^{(m)}$ are simultaneously triagularizable and $\Delta_{1}^{(1)}, \ldots, \Delta_{1}^{(m)}$ are eigen-connections of $A_{1}$ associated with the same simple eigenvalues. If $m=2$, an interconnection of this form appears in hierarchical discretization of a class of distributed parameter systems [17]. If $A_{1}, \Delta_{1}^{(1)}, \ldots, \Delta_{1}^{(m)}$ are circulant matrices and $K_{2}^{(1)}, \ldots, K_{2}^{(m)}$ are defined by

$$
K_{2}^{(k)}=\left(\begin{array}{l|l} 
& I_{n_{2}-1} \\
\hline 1 &
\end{array}\right)^{k},
$$

the resulting interconnection coincides with a model considered in [18]. Furthermore, the intergroup connection matrices $\Delta_{1}^{(1)}, \ldots, \Delta_{1}^{(m)}$ are eigen-connection matrices of $A_{1}$ since all the circulant matrices have the same eigenvector in common.

\section{Conclusion}

In this paper, we have analyzed and designed hierarchical interconnection structures for large-scale multi-agent systems based on the eigenvector. We have defined a class of low rank interlayer information flow that can affect on a few eigenvalues of local interconnection structures selectively. Based on this property, we have developed a procedure for constructing hierarchical interconnections that result in desirable eigenvalue distributions. Whereas large-scale networks are usually modeled as high-dimensional matrices, the proposed procedure enable us to design such a matrix by dealing with a number of lower dimensional matrices. This is a superiority of our method.

We point to two issues as future works. Instead of homogeneous groups, the case where the numbers of agents in each groups are different is more realistic. This is an ongoing topic and is partly tackled in [19]. An extension to the multi-layer case is also interesting.

\section{Acknowledgment}

This work was supported in part by Grant-in-Aid for JSPS Fellows (21.7445).

\section{Appendix A. Proof of Theorem 1}

In some related works $[13,20,21]$, the matrix of the form in (3) can be similarity-transformed to a block upper triangular matrix. Indeed, let the Schur form of $K_{2}$ be given by $U K_{2} U^{*}$, that is, $U$ is a unitary matrix and $U K_{2} U^{*}$ is an upper triangular matrix whose diagonal entries are the eigenvalues of $K_{2}$. Then, $U \otimes I_{n_{1}}$ is a unitary matrix and we have

$$
\left(U \otimes I_{n_{1}}\right) A\left(U^{*} \otimes I_{n_{1}}\right)=I_{n_{2}} \otimes A_{1}+\left(U K_{2} U^{*}\right) \otimes \Delta_{1} .
$$

This implies that the set of all the eigenvalues of $A$ is given by

$$
\sigma(A)=\bigcup_{\gamma \in \sigma\left(K_{2}\right)} \sigma\left(A_{1}+\gamma \Delta_{1}\right) .
$$

Hence, we only have to derive the eigenvalue of $A_{1}+\gamma \Delta_{1}$.

If $\Delta_{1}$ is a right eigen-connection matrix of $A_{1}, \Delta_{1}^{*}$ is a left eigen-connection matrix of $A_{1}^{*}$. Thus, we prove the theorem only for left eigen-connection matrices. Since $\Delta_{1}=\mu \zeta^{*}$ is a left eigen-connection matrix, $\zeta$ is a left eigenvector of $A_{1}$. Left multiplying $A_{1}+\gamma \Delta_{1}$ by $\zeta^{*}$, we obtain the following relation:

$$
\zeta^{*}\left(A_{1}+\gamma \Delta_{1}\right)=\left(\lambda_{1}+\gamma \zeta^{*} \mu\right) \zeta^{*} .
$$

This means that $\zeta$ is a left eigenvector of $A_{1}+\gamma \Delta_{1}$ associated with an eigenvalue $\lambda_{1}+\gamma \zeta^{*} \mu$.

We show the remaining eigenvalues. Let $w_{2}, \ldots, w_{n}$ be (generalized) right eigenvectors of $A_{1}$ associated with all the eigenvalues except $\lambda_{1}$. Since $\zeta$ is a left eigenvector of $A_{1}$ and a left eigenvector is orthogonal to right (generalized) eigenvectors except the corresponding one, we have $\zeta^{*} w_{j}=0$ for all $j=2, \ldots, n$. This fact implies that

$$
\Delta_{1} w_{j}=\mu \zeta^{*} w_{j}=0
$$

which yields the following relation:

$$
\left(A_{1}+\gamma \Delta_{1}\right) w_{j}=A_{1} w_{j}, \quad j=2, \ldots, n .
$$

This means that $w_{2}, \ldots, w_{n}$ are also (generalized) right eigenvectors of $A_{1}+\gamma \Delta_{1}$ and, thus, we have

$$
\sigma\left(A_{1}\right) \backslash\left\{\lambda_{1}\right\}=\sigma\left(A_{1}+\gamma \Delta_{1}\right) \backslash\left\{\lambda_{1}+\gamma \zeta^{*} \mu\right\} .
$$

This completes the proof.

\section{Appendix B. Proof of Theorem 2}

As in the proof of Theorem 1, we consider the eigenvalues of $A_{1}+\gamma \Delta_{1}$ only.

Let $\lambda_{3}, \ldots, \lambda_{n}$ be all the eigenvalues of $A_{1}$ except $\lambda_{1}$ and $\lambda_{2}$, where they are not necessary distinct. Denote (generalized) right eigenvectors of $A_{1}$ associated with $\lambda_{3}, \ldots, \lambda_{n}$ by $w_{3}, \ldots, w_{n}$, respectively. Then, we have $\Delta_{1} w_{j}=0$ for all $j=3, \ldots, n$, because $\zeta_{1}$ and $\zeta_{2}$ are right eigenvectors of $A_{1}$. Thus, we obtain

$$
\left(A_{1}+\gamma \Delta_{1}\right) w_{j}=A_{1} w_{j}, \quad j=3, \ldots, n .
$$

This fact implies that $w_{j}$ is also a right eigenvector of $A_{1}+\gamma \Delta_{1}$. Thus, the following relation holds:

$$
\sigma\left(A_{1}\right) \backslash\left\{\lambda_{1}, \lambda_{2}\right\}=\left\{\lambda_{3}, \ldots, \lambda_{n}\right\} \subset \sigma\left(A_{1}+\gamma \Delta_{1}\right) .
$$


Let $\lambda_{1}^{\prime}$ and $\lambda_{2}^{\prime}$ be the remaining eigenvalues of $A_{1}+\gamma \Delta_{1}$. We assume that $\lambda_{1}^{\prime}, \lambda_{2}^{\prime} \notin \sigma\left(A_{1}\right) \backslash\left\{\lambda_{1}, \lambda_{2}\right\}$. Then, the eigenvectors associated with $\lambda_{1}^{\prime}$ and $\lambda_{2}^{\prime}$ must be included in the subspace spanned by $v_{1}$ and $v_{2}$, eigenvectors of $A_{1}$ associated with $\lambda_{1}, \lambda_{2}$, because they are included in $\operatorname{span}\left(w_{3}, \ldots, w_{n}\right)^{\perp}$. Let $v_{1}^{\prime}$ and $v_{2}^{\prime}$ be the left eigenvectors of $A_{1}+\gamma \Delta_{1}$ associated with $\lambda_{1}^{\prime}$ and $\lambda_{2}^{\prime}$, respectively, and they are represented by

$$
v_{l}^{\prime}=\alpha_{l 1} v_{1}+\alpha_{l 2} v_{2}, \quad l=1,2 .
$$

Left-multiplying $A_{1}+\gamma \Delta_{1}$ by $v_{l}^{\prime}$ yields

$$
\begin{aligned}
& v_{l}^{\prime *}\left(A_{1}+\gamma \Delta_{1}\right)=\lambda_{l}^{\prime} v_{l}^{* *} \\
&\left(\begin{array}{l}
\alpha_{l 1} \\
\alpha_{l 2}
\end{array}\right)^{*}\left(\begin{array}{c}
v_{1}^{*} \\
v_{2}^{*}
\end{array}\right)\left(A_{1}+\gamma\left(\begin{array}{ll}
\mu_{1} & \mu_{2}
\end{array}\right) T^{*}\left(\begin{array}{c}
v_{1}^{*} \\
v_{2}^{*}
\end{array}\right)\right)=\lambda_{l}^{\prime}\left(\begin{array}{l}
\alpha_{l 1} \\
\alpha_{l 2}
\end{array}\right)^{*}\left(\begin{array}{c}
v_{1}^{*} \\
v_{2}
\end{array}\right) \\
&\left(\begin{array}{l}
\alpha_{l 1} \\
\alpha_{l 2}
\end{array}\right)^{*}\left(\left(\begin{array}{ll}
\lambda_{1} & \\
& \lambda_{2}
\end{array}\right)+\gamma\left(\begin{array}{l}
v_{1}^{*} \\
v_{2}^{*}
\end{array}\right)\left(\begin{array}{ll}
\mu_{1} & \mu_{2}
\end{array}\right) T^{*}\right)\left(\begin{array}{c}
v_{1}^{*} \\
v_{2}^{*}
\end{array}\right) \\
&=\lambda_{l}\left(\begin{array}{l}
\alpha_{l 1} \\
\alpha_{l 2}
\end{array}\right)^{*}\left(\begin{array}{l}
v_{1}^{*} \\
v_{2}^{*}
\end{array}\right) .
\end{aligned}
$$

Hence, we have

$$
\left(\begin{array}{c}
\alpha_{l 1} \\
\alpha_{l 2}
\end{array}\right)^{*}\left(\Phi_{\gamma}-\lambda_{l} I_{2}\right)\left(\begin{array}{l}
v_{1}^{*} \\
v_{2}^{*}
\end{array}\right)=0 .
$$

Since $v_{1}(\neq 0)$ and $v_{2}(\neq 0)$ are linearly independent, $\alpha_{l 1}, \alpha_{l 2}$, and $\lambda_{l}$ must satisfy

$$
\left(\begin{array}{c}
\alpha_{l 1} \\
\alpha_{l 2}
\end{array}\right)^{*}\left(\Phi_{\gamma}-\lambda_{l} I_{2}\right)=0 .
$$

Furthermore, $\left(\alpha_{l 1}, \alpha_{l 2}\right) \neq 0$ due to $v_{l}^{\prime} \neq 0$. These facts mean that $\lambda_{l}$ is an eigenvalue of $\Phi_{\gamma}$ and that $\left(\alpha_{l 1}, \alpha_{l 2}\right)^{\top}$ is the corresponding left eigenvector. If $\Phi_{\gamma}$ has two distinct eigenvalues or one repeated eigenvalue that has geometric multiplicity 2 , then $u_{l}^{\prime}$ 's are eigenvectors of $A_{1}+\gamma \Delta_{1}$. Otherwise, the last eigenvalue of $A_{1}+\gamma \Delta_{1}$ still remains. However, this case corresponds to the case where one of $\lambda_{1}^{\prime}$ and $\lambda_{2}^{\prime}$ agrees with one of the elements in the set $\sigma\left(A_{1}\right) \backslash\left\{\lambda_{1}, \lambda_{2}\right\}$. This completes the proof.

\section{References}

[1] W. Ren, R. W. Beard, E. M. Atkins, A survey of consensus problems in multi-agent coordination, in: Proc. Americal Control Conference, 2005, pp. 1859-1864.

[2] R. Olfati-Saber, J. A. Fax, R. M. Murray, Consensus and cooperation in networked multi-agent systems, Proceedings of the IEEE 97 (1) (2007) 215-233.

[3] R. M. Murray, Recent research in cooperative control of multivehicle systems, J. Dyn. Sys., Meas., Control 129 (5) (2007) 571-583.

[4] M. Mesbahi, M. Egerstedt, Graph Theoretic Methods in Multiagent Networks, Princeton University Press, 2010.

[5] E. Ravasz, A. L. Somera, D. A. Mongru, Z. N. Oltvai, A.-L. Barabási, Hierarchical organization of modularity in metabolic networks, Science 297 (2002) 1551-1555.

[6] S. L. Smith, M. E. Broucke, B. A. Francis, A hierarchical cyclic pursuit scheme for vehicle networks, Automatica 41 (2005) 1045-1053.

[7] S. C. Hamilton, M. E. Broucke, Patterned linear systems: Rings, chains, and trees, in: 49th IEEE Conference on Decision and Control, 2010, pp. 1397-1402.
[8] S. C. Hamilton, M. E. Broucke, Geometric control of patterned linear systems, in: 49th IEEE Conference on Decision and Control, 2010, pp. 1403-1408.

[9] H. Shimizu, S. Hara, Cyclic pursuit behavior for hierarchical multi-agent systems with low-rank interconnection, in: Proc. SICE Annual Conference, 2008, pp. 3131-3136.

[10] H. Shimizu, S. Hara, Hierarchical consensus for multi-agent systems with low-rank interconnection, in: Proc. ICCAS-SICE, 2009, pp. 1063-1067.

[11] S. Hara, H. Shimizu, T.-H. Kim, Consensus in hierarchical multi-agent dynamical systems with with low-rank interconnections: Analysis of stability and convergence rates, in: Proc. American Control Conference, 2009, pp. 5192-5197.

[12] A. Williams, S. Glavaški, T. Samad, Formations of formations: hierarchy and stability, in: Proc. American Control Conference, 2004, pp. 29922997.

[13] P. Massioni, M. Verhaegen, Distributed control for identical dynamically coupled systems: a decomposition approach, IEEE Trans. Autom. Control 54 (1) (2009) 124-135.

[14] S. Hara, T. Hayakawa, H. Sugata, LTI systems with generalized frequency variables: A unified framework for homogeneous multi-agent dynamical systems, SICE JCMSI 2 (5) (2009) 299-306.

[15] H. Tanaka, S. Hara, T. Iwasaki, LMI stability condition for linear systems with generalized frequency variables, in: Proc. the 7th Asian Control Conference, 2009, pp. 136-141.

[16] D. S. Bernstein, Matrix Mathematics: Theory, Facts, and Formulas with Application to Linear Systems Theory, Princeton University Press, 2005.

[17] D. Tsubakino, S. Hara, Hierarchical modeling for diffusion systems: Symmetrically-networked systems with rank one interconnection, in: Proc. ICCAS-SICE, 2009, pp. 1068-1073.

[18] Y. Wang, M. Morari, Structure of hierarchical linear systems with cyclic symmetry, Systems \& Control Letters 58 (2009) 241-247.

[19] N. Fujimori, L. Liu, S. Hara, D. Tsubakino, Hierarchical network synthesis for output consensus by eigenvector-based interlayer connections, in: 50th IEEE Conference on Decision and Control and European Control Conference, 2011.

[20] J. A. Fax, R. M. Murray, Information flow and cooperative control of vehicle formations, IEEE Trans. Autom. Control 49 (9) (2004) 1465-1476.

[21] F. Borrelli, T. Keviczky, Distributed LQR design for identical dynamically decoupled systems, IEEE Trans. Autom. Control 53 (8) (2008) 19011912 . 